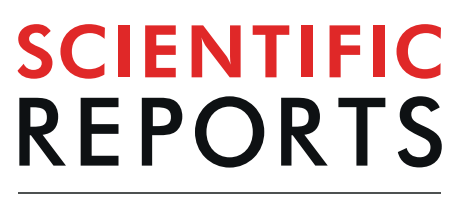

natureresearch

There are amendments to this paper

\title{
R-loop-forming Sequences Analysis in Thousands of Viral Genomes Identify A New Common Element in Herpesviruses
}

\author{
Thidathip Wongsurawat ${ }^{1 *}$, Arundhati Gupta ${ }^{2,3}$, Piroon Jenjaroenpun ${ }^{1}$, Shana Owens ${ }^{2}$, \\ James Craig Forrest ${ }^{2} \&$ Intawat Nookaew ${ }^{1 *}$
}

R-loops are RNA-DNA hybrid sequences that are emerging players in various biological processes, occurring in both prokaryotic and eukaryotic cells. In viruses, R-loop investigation is limited and functional importance is poorly understood. Here, we performed a computational approach to investigate prevalence, distribution, and location of R-loop forming sequences (RLFS) across more than 6000 viral genomes. A total of 14637 RLFS loci were identified in 1586 viral genomes. Over $70 \%$ of RLFS-positive genomes are dsDNA viruses. In the order Herpesvirales, RLFS were presented in all members whereas no RLFS was predicted in the order Ligamenvirales. Analysis of RLFS density in all RLFS-positive genomes revealed unusually high RLFS densities in herpesvirus genomes, with RLFS densities particularly enriched within repeat regions such as the terminal repeats (TRs). RLFS in TRs are positionally conserved between herpesviruses. Validating the computationally-identified RLFS, R-loop formation was experimentally confirmed in the TR and viral Bcl-2 promoter of Kaposi sarcomaassociated herpesvirus (KSHV). These predictions and validations support future analysis of RLFS in regulating the replication, transcription, and genome maintenance of herpesviruses.

Recent advances in the field of genomics have revealed widespread occurrence of non-canonical nucleic acid-forming structures, such as Z-DNA ${ }^{1}$, hairpin loops ${ }^{2,3}$, and G-quadruplexes (guanine-rich sequences that attain specific four-stranded conformations $)^{4}$, in various genomes including viruses ${ }^{5}$. These structures likely play important roles in the replication strategies used by a particular virus. For instance, Z-DNA structures in the simian virus 40 enhancer regions activate transcription ${ }^{1}$. Hairpin structures at the termini of the adeno-associated virus genome promote persistent DNA circles and concatemers during recombination processes that occur in the infected host cell ${ }^{2,3}$. G-quadruplexes are present in several viral genomes e.g., human immunodeficiency virus (HIV- 1), Epstein-Barr virus (EBV), and human papillomavirus (HPV) (reviewed in ${ }^{4}$ and function in various aspects of the replication cycles of these viruses.

Another non-canonical nucleic acid structure called an R-loop or RNA:DNA structure is preferentially formed within G-rich sequences and possesses greater thermodynamic stability than the original DNA:DNA duplex $^{6}$. R-loops have been experimentally observed in a wide range of organisms, from bacteria to mammals ${ }^{6,7}$, where they function in transcription ${ }^{8}$, telomere maintenance ${ }^{9}$, genome instability ${ }^{10,11}$, and epigenetic regulation ${ }^{12}$. They are also associated with certain diseases, such as Prader-Willi syndrome ${ }^{13}$, ataxia with oculomotor apraxia ${ }^{14}$, amyotrophic lateral sclerosis ${ }^{15}$, spinal muscular atrophy ${ }^{16,17}$, motor neuron disorders ${ }^{18}$, cancers ${ }^{19}$ and many oth$\mathrm{ers}^{20}$. R-loops, have been predicted by computational approaches in various organisms ${ }^{21-23}$ and demonstrated by direct experimental evidence, however, genome-scale identification of R-loops in the viral species has not been explored.

Knowledge regarding roles for R-loops in viruses is limited. In 2011, Rennekamp et.al. demonstrated the persistence of R-loops at the origin of replication of Epstein-Barr virus (EBV) ${ }^{24}$. Removal of R-loops by RNase

${ }^{1}$ Department of Biomedical Informatics, College of Medicine, University of Arkansas for Medical Sciences, Little Rock, Arkansas, USA. ${ }^{2}$ Department of Microbiology and Immunology and Center for Microbial Pathogenesis and Host Inflammatory Responses, University of Arkansas for Medical Sciences, Little Rock, Arkansas, USA. ${ }^{3}$ Present address: Department of Pediatrics, University of Pittsburgh School of Medicine, Pittsburgh, PA, USA. *email: twongsurawat@uams.edu; inookaew@uams.edu 
$\mathrm{H}$, an RNA-degrading enzyme specific for RNA-DNA duplex molecules, eliminated the generation of ssDNA at the viral origin, thereby inhibiting viral replication by preventing the recruitment of the ssDNA binding protein BALF2 to the origin of viral replication ${ }^{24}$. In 2014, Jackson et al. demonstrated that R-loops formed during infection by Kaposi sarcoma-associated herpesvirus (KSHV) correlated with a host-cell DNA damage response and genome instability ${ }^{25}$. The mechanism for this likely involves KSHV ORF57, a protein that hijacks the host hTREX complex, an RNA binding protein complex that normally prevents R-loop formation, leading to enhanced R-loop formation and DNA damage in KSHV-infected cells. Although, this study did not report R-loop forming loci in the KSHV genome, it provides important evidence that R-loop formation in virus-infected cells might be impacted by the virus life cycle. Notably, both studies highlighted the investigation of R-loop formation in herpesviruses.

In this study, we employed our previously developed computer algorithm, QmRLFS-finder ${ }^{26}$, to identify possible R-loop forming sequences (RLFSs) in a reference set of over 6000 viral genomes. The genome set was retrieved from the National Center for Biotechnology Information (NCBI) database and exhibits remarkable diversity in length and information content ${ }^{27}$ across a wide range of viral families. We found that approximately $25 \%$ of the total dataset contains at least one RLFS in a specific genome with an expected overrepresentation in dsDNA viruses compared to other virus types, especially in the Herpesviridae order. The distribution and association of RLFS in herpesvirus genomes and unique genomic features including coding, non-coding regions, and repeat regions were further investigated and experimentally validated to highlight potential roles for R-loops in the herpesvirus life cycle.

\section{Materials and Methods}

Data acquisition and data availability. We used a set of viral genomes available on $17^{\text {th }}$ Jan, 2017 from NCBI (http://www.ncbi.nlm.nih.gov). We retrieved the DNA sequences (for RNA viruses, NCBI provide DNA sequences version of the RNA genome) of the genomes from the database and preprocessed following Zhang et al. ${ }^{27}$, obtaining a total of 6153 viruses that will be used in further steps. Viral taxonomic classification data (i.e., The Baltimore classification and the International Committee on Taxonomy of Viruses or ICTV classification) was also collected from NCBI taxonomy database (http://www.ncbi.nlm.nih.gov/taxonomy) ${ }^{28}$ and used for further analysis and interpretations.

R-loop forming sequence (RLFS) prediction by QmRLFS-finder. We predicted possible R-loop forming structures on both strands of genomic DNA/RNA using our QmRLFS-finder software ${ }^{26}$. Briefly, QmRLFS-finder identify RLFS based on three structural features in the sequence beginning with a short G-cluster sequence is responsible for initiating R-loop formation (R-loop initiation zone), then a linker sequences that connect to a downstream region of G-rich sequence as called R-loop elongation. The predicted RLFSs that are overlapped at least one nucleotide were then merged together to be one RLFS. The number of RLFSs found in each genome was reported and available in Supplementary Table 3. The RLFSs located in the coding sequence (CDS) and non-CDS are identified using BEDtools $2^{29}$. Visualization of the predicted RLFS results and statistical analysis were performed under R suite software (https://www.r-project.org/) and Bio-Graphics module (https:// metacpan.org/release/Bio-Graphics) in perl language.

Cell culture and virus. The iSLK and iSLK-BAC16 cells, which are derived from human endothelial cells, were a gift from Dr. Jae Jung ${ }^{30}$. The cells were cultured in Dulbecco's Modified Eagle's Medium supplemented with $10 \%$ FBS, $1 \% \mathrm{~L}-\mathrm{Gln}, 100 \mathrm{U} / \mathrm{ml}$ of penicillin, $100 \mathrm{U} / \mathrm{ml}$ of streptomycin, $250 \mu \mathrm{g} / \mathrm{ml}$ of geneticin, and $1 \mu \mathrm{g} / \mathrm{ml}$ of puromycin. $1200 \mu \mathrm{g} / \mathrm{ml}$ hygromycin B was added to iSLKs-BAC16 culture media to maintain KSHV. Cells were cultured at $37^{\circ} \mathrm{C}$ with $5 \% \mathrm{CO}_{2}$ and $\sim 99 \%$ humidity. KSHV lytic replication was induced by replacing culture media with DMEM supplemented with $10 \% \mathrm{FBS}, 100 \mathrm{U} / \mathrm{ml}$ penicillin, $100 \mu \mathrm{g} / \mathrm{ml}$ streptomycin, $2 \mathrm{mM} \mathrm{L}$-glutamine, $1 \mu \mathrm{g} /$ $\mathrm{ml}$ doxycycline and $1 \mathrm{mM}$ sodium butyrate. Cells were induced for 48 hours. Induction of the lytic cycle was confirmed by quantitative PCR for the KSHV genome, which revealed a $>30$-fold increase in viral genomes relative to cellular genomes by $48 \mathrm{~h}$ post-induction.

Extraction of nuclear materials. A total of $10^{7}$ iSLK or iSLK-BAC16 cells were lysed in $500 \mu l$ lysis buffer $(0.5 \% \mathrm{SDS}, 50 \mathrm{mM} \mathrm{NaCl}, \mathrm{pH} 8.0)$ with 1 unit of proteinase $\mathrm{K}$ (New England Biolabs or NEB) at $37^{\circ} \mathrm{C}$ overnight. Total nucleic acids were precipitated with $1.5 \mathrm{M} \mathrm{NaCl}$ and isopropanol, and resuspended in $500 \mu \mathrm{l} \mathrm{TE} \mathrm{buffer.} 50 \mu \mathrm{g}$ of genomic DNA was digested using a mixture containing 10 units each of XbaI, XhoI, EcoRI and BamHI (NEB) in CutSmart Buffer (NEB) at $37^{\circ} \mathrm{C}$ overnight. Each sample was split into two, and one half was digested with RNase $\mathrm{H}$ (NEB) at $37^{\circ} \mathrm{C}$ for 8 hours. Nucleic acids were purified from all samples by phenol:chloroform extraction followed by ethanol precipitation. Purified material was resuspended in IP buffer ( $50 \mathrm{mM}$ Tris-Cl, $150 \mathrm{mM}$ $\mathrm{NaCl}, 1 \% \mathrm{NP}-40$, pH 8.0).

DNA-RNA Immunoprecipitation (DRIP). DRIP was performed essentially as previously described with minor modifications ${ }^{31}$. In brief, $10 \mu \mathrm{g}$ of DNA was incubated with $4 \mu \mathrm{g}$ of S9.6 (Kerafast) or isotype control (mouse $\mathrm{IgG}$ ) in $500 \mu \mathrm{l} \mathrm{IP} \mathrm{buffer}$ at $4{ }^{\circ} \mathrm{C}$ overnight. $30 \mu \mathrm{l}$ of Dynabeads Protein G beads (Thermo Fisher Scientific) was added to each sample, and samples were rotated for $1 \mathrm{~h}$ at room temperature. Antibody-DNA-RNA complexes were pelleted and were eluted in $200 \mu \mathrm{l}$ of elution buffer provided in the kit. Precipitation complexes were digested for $2 \mathrm{~h}$ with proteinase $\mathrm{K}$, and nucleic acids were purified by phenol:chloroform extraction followed by ethanol precipitation. Recovered fragments were analyzed by PCR and qPCR.

Drip-PCR. Samples were analyzed for the presence of two predicted RLFS in the KSHV genome at the ORF16 promoter and the terminal repeat (TR). A previously defined $c-M Y C$ RLFS was used as a positive control to ensure successful DRIP ${ }^{19}$. Primer pairs were designed with Primer-BLAST ${ }^{32}$. A 206nt segment within ORF45 

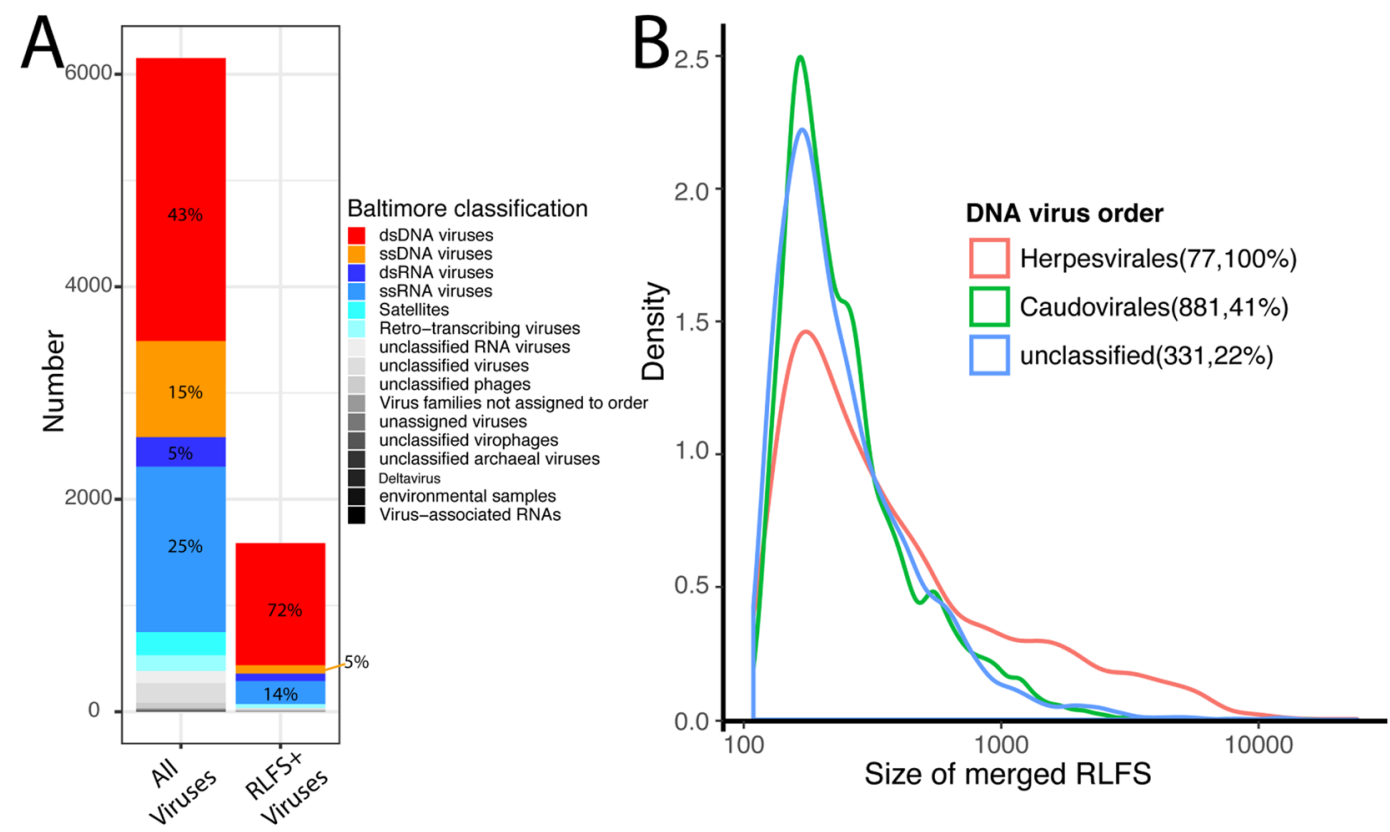

Figure 1. Distribution RLFS-positive genome in viral kingdom. (A) Bar chart shows the distribution of all viral genomes in the dataset with the RLFS-positive genomes. The colors represent the different group of viruses based on Baltimore classification. (B) Density plots show the distribution of size of detected merged RLFS in different order level of DNA virus based on ICTV classification. The numbers and percentage values in parenthesis represent the number of RLFS-positive genome and percentage RLFS-positive genome of for each order, respectively.

(nt 67600 to 67805 in NC009333.1) served as a negative control for R-loops, as there are no predicted RLFS at this locus. PCR was performed using Hot Start 2x Taq mastermix (NEB) on an Applied Biosystems SimpliAmp thermal cycler (Thermo Fisher Scientific) using the following reaction parameters: $5 \mathrm{~min}$ at $95^{\circ} \mathrm{C}$ followed by 30 cycles of $15 \mathrm{~s}$ at $95^{\circ} \mathrm{C}, 15 \mathrm{~s}$ at $60^{\circ} \mathrm{C}$, and $30 \mathrm{~s}$ at $72^{\circ} \mathrm{C}$. PCR products were resolved by electrophoresis on $1.2 \%$ agarose. Primer pairs were as follows. ORF16-promoter: forward $5^{\prime}$-ACATTTGCCCCACCGTCGCCT-3'; reverse 5'- GCACATAGCACGCGCACAGCA-3'. Terminal repeat: forward 5'-GACCCCGGGCAGCGAGGGAA-3'; reverse 5'-AGGGCTCCACGTAGCAAGCACTG-3'. ORF45 (absence of predicted RLFS): forward 5'-GGGAGGTGACCCTTTGTGCT-3'; reverse 5'-CTCATGGACGTGGGCCAGA-3'.

qPCR. Genome copy number was determined by qPCR relative to uninduced iSLK-BAC16 samples to verify induction of the KSHV lytic cycle in iSLK-BAC16 cells treated with doxycycline and sodium butyrate. Quantitative PCR was performed with $\mathrm{RT}^{2}$ SYBR Green ROX qPCR mastermix (Qiagen) on an Applied Biosystems QuantStudio 6 Flex thermal cycler (Thermo Fisher Scientific) using the following reaction parameters: $10 \mathrm{~min}$ at $95^{\circ} \mathrm{C}$ followed by $40 \mathrm{cycles}$ of $15 \mathrm{~s}$ at $95^{\circ} \mathrm{C}$ and $1 \mathrm{~min}$ at $60^{\circ} \mathrm{C}$. Data were analyzed using the percentage of input method where $\Delta \mathrm{Ct}=\mathrm{Ct}_{\text {input }}{ }^{-} \mathrm{Ct}_{\mathrm{IP} \text {. }}$ Results are expressed as $100^{*}\left(2^{\Delta \mathrm{Ct}}\right)$.

\section{Results}

R-loop forming sequences are predominant in dsDNA viruses. We analyzed a total of 6153 viral genomes, which are 2663 double-stranded DNA (dsDNA), 1556 single-stranded RNA (ssRNA), 905 single-stranded DNA (ssDNA), 278 double-stranded RNA (dsRNA), and 751 other viruses based on the Baltimore classification scheme (Supplementary Table 1). We predicted RLFSs and merged them if they overlapped to each other (at least one nucleotide). All RLFSs which passed merging process were called merged RLFS (mRLFS). From the dataset, we identified 14637 mRLFS found in 1586 genomes. From these genomes, 1148 genomes were dsDNA (72\% of total) followed by $216(14 \%), 78(5 \%)$, and 71 (4\%) in ssRNA, ssDNA, and dsRNA, respectively (Fig. 1A). Even though R-loop formation in the genome of ssRNA and dsRNA viruses is not logically possible, the predicted results may be useful for study of RNA viruses that have RNA-DNA hybrid structures during their replication (e.g. human immunodeficiency virus type 1$)^{33}$. We then focused on the genomes of DNA viruses for further analysis. In the reference dataset, there are 3 orders of DNA virus with unclassified order (see Supplementary Table 2); surprisingly, Ligamenvirales, which are linear dsDNA genome viruses known as an archaea infectious agent, had no RLFS-positive genome. In contrast, all viruses in the herpesvirales order, which consists of eukaryotic dsDNA viruses found only in animal hosts, contain predicted RLFS within their genomes, and the size of mRLFS in these viruses was longer than others viral families (Fig. 1B). Very long mRLFS were found in the highly G-rich repetitive regions of herpesviruses (see Fig. 2). The details of the number of RLFS-positive and RLFSnegative genomes in each family are provided in Supplementary Table 3. 

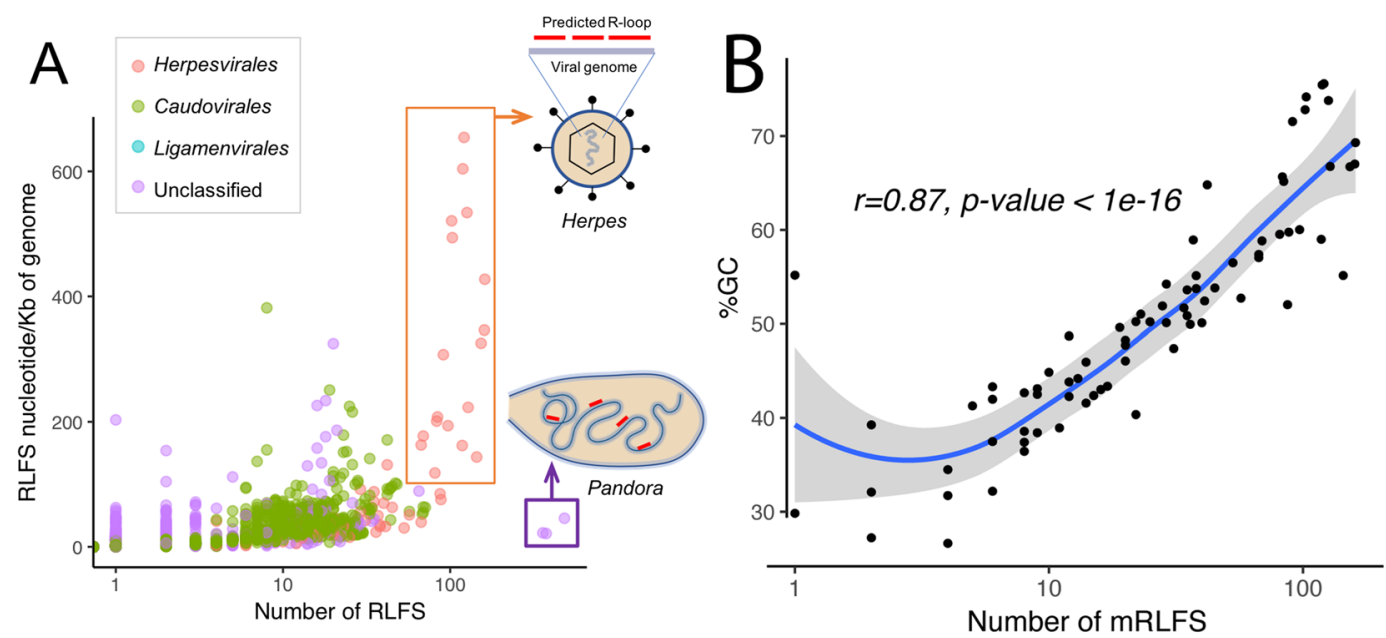

Figure 2. Association between indentified mRLFS number with mRLFS density on the genome or \%GC content. (A) Scatter plot shows mRLFS density (mRLFS nucleotide per kilobase of genome) versus number of mRLFS of individual RLFS-positive genome. Red and magenta box indicate herpesviruses and pandoraviruses, respectively that illustrated in cartoon picture. Blue and red bars represent genome and mRLFS (B) Scatter plot shows strong correlation between \%GC content and number of mRLFS of all 77 herpesvirus genomes in the reference dataset.

Herpesvirus genomes have unusually high RLFS density. A key factor influencing detection of RLFS in any sequence is genome length, which increases the probability of finding RLFS over G-rich regions ${ }^{6,7}$. We therefore investigated the association between number of detected mRLFS relative to viral genome size and GC content for the RLFS-positive genomes of DNA viruses. Across all DNA viral genomes, the giant viruses, Pandoravirus dulcis, $P$. salinus, and P. inopinatum contained the largest number of mRLFS: 482, 375, and 358 mRLFS, respectively (Supplementary Table 4). On the other hand, only one mRLFS was detected in several of very small genomes such as virus satellites. The mRLFS density (mRLFS/kb of sequence) in a given viral genome is plotted as a function of total numbers of mRLFS per virus in Fig. 2A. While pandoraviruses have the largest number of mRLFS in their genomes, this corresponds to only $9 \%$ of their genome length. However, herpesviruses have both high numbers of mRLFS and high coverage of the genome (Fig. 2A). Consistent with the observation that R-loops are highly associated with G-rich sequences ${ }^{6,7}$, we found a strong correlation $(r=0.87$, $p$-value $<1$ e16) between the number of mRLFS and the GC content of herpesvirus genomes as shown in Fig. 2B. We then compared the identified mRLFS in herpesviruses with randomly shuffled DNA sequences $(\mathrm{n}=30)$ from each individual herpesvirus genome that preserved GC content and genome length illustrated in Supplementary Fig. 1. We found that the number of mRLFS identified in herpesviruses was higher than in the random sequences when GC content was below $65 \%$. On the contrary, when GC content was greater than $65 \%$, fewer mRLFS were identified in the herpesviruses than in the randomized sequences. This indicated an evolutionary basis for the existence of non-random GC clusters pattern in herpesvirus genomes.

mRLFS are common in terminal repeat regions of herpesviruses. Herpesviridae is composed of three subfamilies (Alphaherpesvirinae, Betaherpesvirinae, and Gammaherpesvirinae), whose genome sizes range from 120 to $230 \mathrm{kbp}$ and contain approximately 60 to 120 ORFs. We investigated the distribution of mRLFS for individual genomes, including coding sequence (CDS), non-CDS, and repeat regions of all herpesviridae members (Supplementary Fig. 2). We found a strong positive correlation $(\mathrm{r}=0.88)$ between $\mathrm{GC}$ content and the number of mRLFS found in CDS. In contrast, non-CDS exhibited a negative correlation $(\mathrm{r}=-0.57)$ between GC content and the number of mRLFS. However, the distribution pattern of mRLFS in CDS and non-CDS was variable between different herpesviruses (Supplementary Fig. 2). It is thus difficult to comment on the possible functions of Rloop in CDS vs non-CDS.

Of note, our computational-based approach predicted the presence of an RLFS in the Epstein-Barr virus (EBV, also known as human gammaherpesvirus-4) origin of replication (Supplementary Fig. 3). This R-loop was previously reported and demonstrated to facilitate viral DNA replication by enabling binding of BALF2 protein to the origin of replication ${ }^{24}$. This observation provides an independent experimental validation of our prediction tool and provides confidence on the identified RLFS by the computational approach presented here.

Herpesvirus genomes contain repetitive regions, especially at the termini of the linear genomes (terminal repeats, TR) that play crucial regulatory roles in genome maintenance ${ }^{34}$. Mapping mRLFS on repetitive regions of representative genomes from each subfamily (Fig. 3) revealed a high degree of overlap between mRLFS and the $3^{\prime}$ TR that were conserved across the different subfamilies (see Supplementary Fig. 4 for all 77 genomes). The sequence patterns of mRLFS predicted in the $3^{\prime}$ TRs of herpesvirus genomes resemble those of experimentally determined mRLFS found in mammalian immunoglobulin switch regions, which is also an R-loop prone region (Supplementary Fig. 5) ${ }^{35}$. In addition, some herpesvirus genomes have TTAGGG repeats identical to host cell telomere repeats ${ }^{36,37}$. R-loops have also been detected in the telomere repeat elements of host organisms and 
A

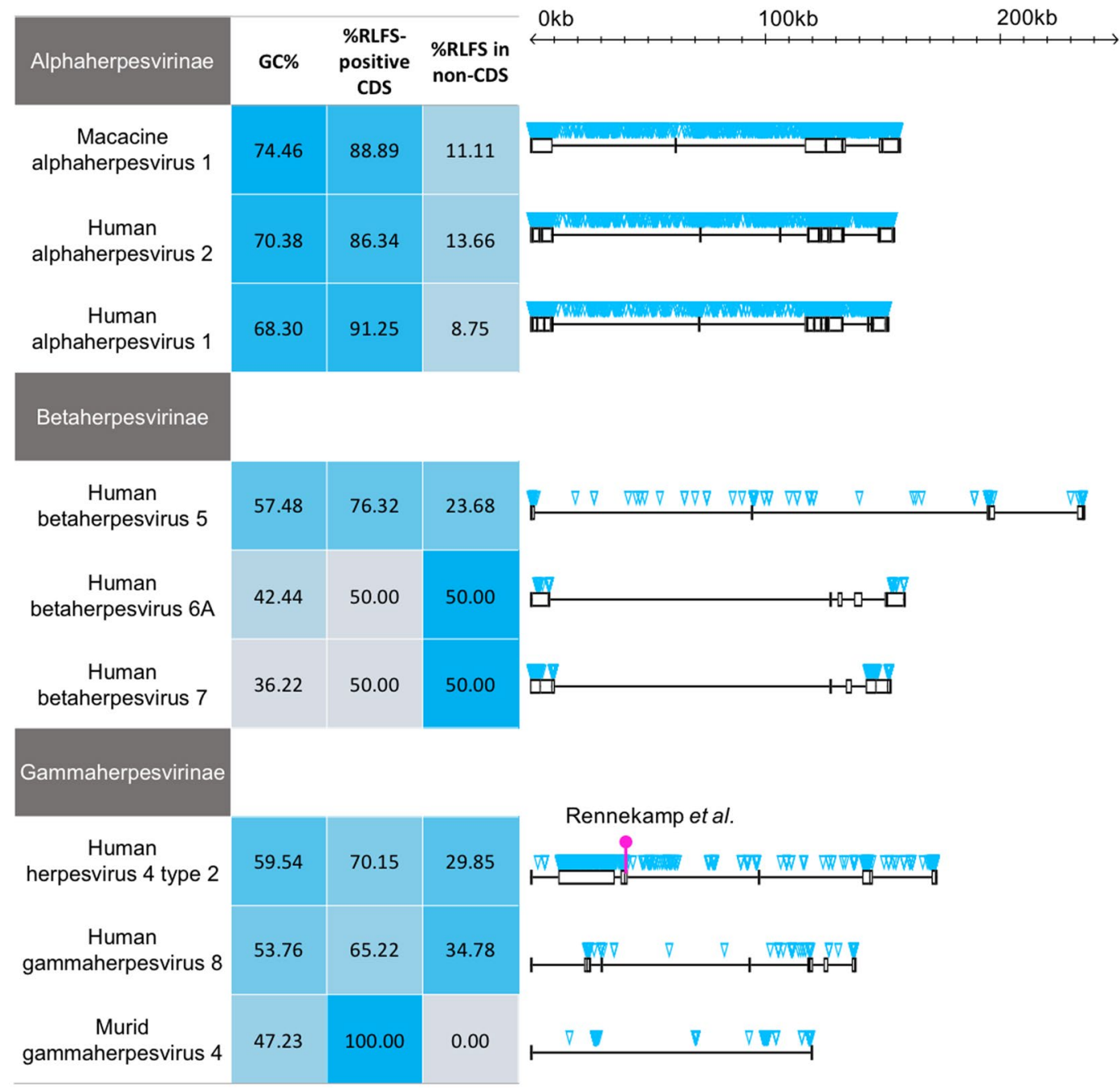

Figure 3. Schematic representation of the distribution of mRLFS in selected representative herpesviruses in each subfamily. (A) Heat map plots. From the left to the right column represent percent of GC content, percent of mRLFS found in CDS and percent of mRLFS found in non-CDS, respectively. (B) Distribution of mRLFS in the genome. The genomes were drawn to scale. The black line represented the genomes with open boxes represent the repeat regions in the genome. The blue triangles represent the genomic positions of mRLFS. The magenta mark is the previously known R-loop forming locus on the Epstein-Barr virus (EBV) genome ${ }^{24}$.

have an essential role in telomere maintenance ${ }^{34}$. Similar to host genomes, herpesvirus TRs are essential for viral genome stability ${ }^{38}$. This raises the question whether herpesvirus R-loops serve the same function as host R-loops.

Experimental validation of select RLFS loci in the KSHV genome. To experimentally validate the newly predicted mRLFS in herpesvirus genomes, we evaluated select genomic regions of the Kaposi sarcoma-associated herpesvirus (KSHV, also known as human gammaherpesvirus-8). Two predicted RLFS-positive regions, the promoter for ORF16 (viral Bcl2) and within the TR, were selected for validation based on their published importance in KSHV infection (Fig. 4A). A third region that corresponded to ORF45 (nucleotide 67600 to 67805 in the NC009333.1 KSHV genome sequence) was not predicted to encode RLFS and was chosen as a negative control. We performed DNA:RNA immunoprecipitation (DRIP) using antibody S9.6, which specifically recognizes DNA:RNA hybrid molecules, on (i) uninfected iSLK cells, (ii) induced uninfected iSLK cells (iii) iSLK-BAC16 cells latently infected with KSHV and (iv) induced iSLK-BAC16 cells undergoing lytic viral replication. As specificity controls, parallel DRIP was performed on isolated nucleic acids treated with RNase $\mathrm{H}$ to degrade DNA:RNA hybrids. A previously identified RLFS in the cellular $c-M Y C$ promoter was evaluated as a positive control for DRIP. For ORF16 and $c-M Y C$, quantitative PCR (qPCR) demonstrated superior precipitation in samples not treated with RNase $\mathrm{H}$ in comparison to RNase $\mathrm{H}$ digested samples, while the negative control ORF45 sequence was only minimally detected in S9.6 precipitates (Fig. 4B). These data therefore confirmed the presence of an RLFS in the ORF16 promoter region of the KSHV genome. Interestingly, the viral RLFS tested were detected in both latently infected cells and cells undergoing lytic replication (Fig. 4B), suggesting that RLFS are 


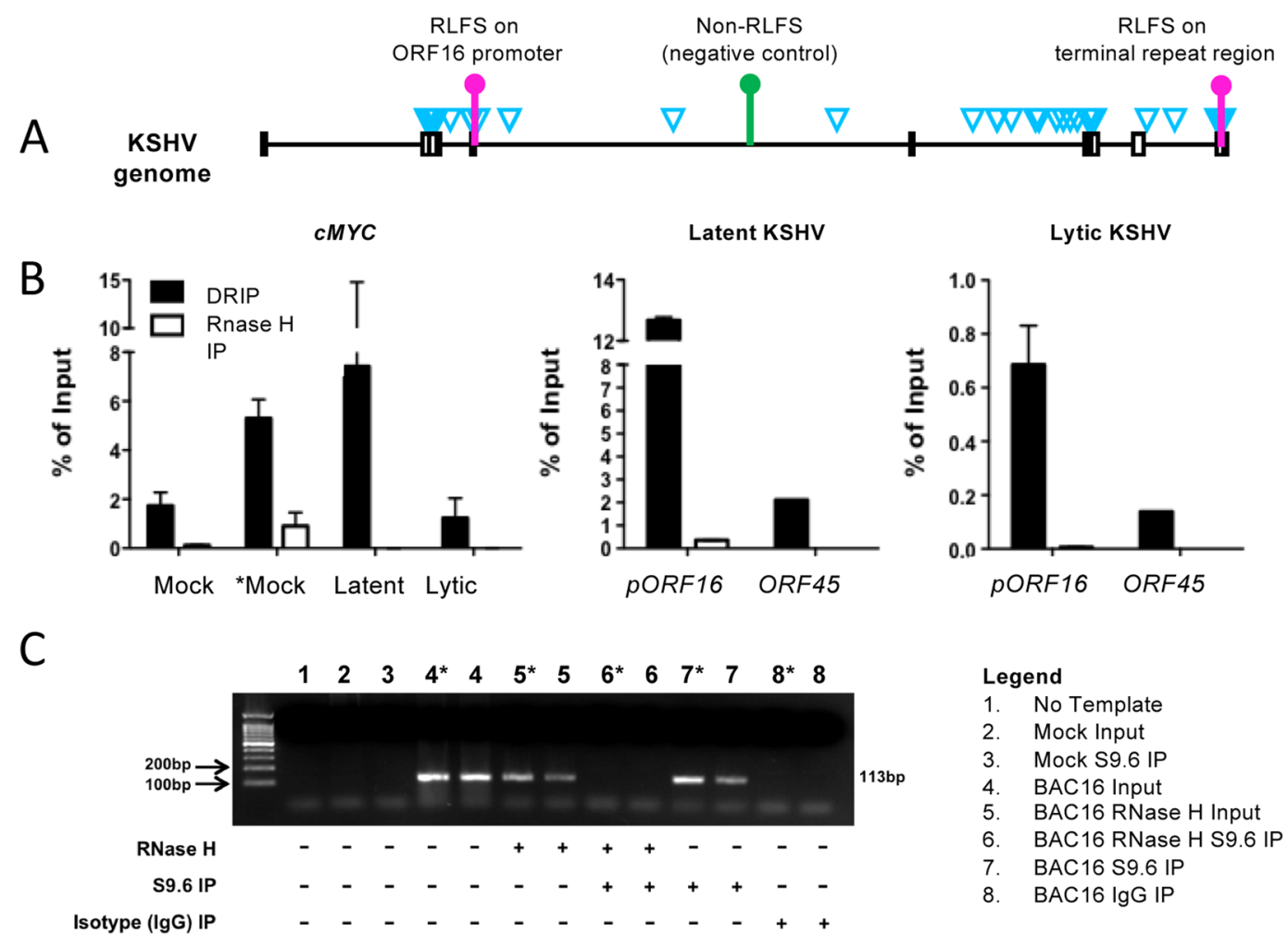

Figure 4. R-loop formation in Kaposi sarcoma-associated herpesvirus (KSHV) genome. Total nucleic acid from iSLK cells latently infected with KSHV, undergoing lytic viral replication, and uninfected cells (mock) were subjected DNA-RNA immunoprecipitation with $\mathrm{S9.6}$ antibody. RNase H digestion was used as a negative control to ensure S9.6 was specific for DNA-RNA hybrids. A mouse IgG served as an isotype control to ensure DNA-RNA binding to S9.6 was specific. (A) Schematic representation of KSHV genome. Selected loci of RLFS and non-RLFS for experimental validation are shown in pink and green mark. The blue triangles represent the positions of RLFS. The boxes represent the repeat regions in the genome. (B) qPCR was performed to measure specific immunoprecipitation of KSHV ORF16 promoter by S9.6, cMYC was used as a positive control, and a region of KSHV ORF45 was used as a negative control. RNase $\mathrm{H}$ digestion was used as a treatment control to ensure S9.6 was specific for DNA:RNA hybrids. (C) R-loops were detected in the terminal repeat region of KSHV genomes by PCR. *indicates lytic BAC16. Reactivated (lytic) samples were treated with sodium butyrate and doxycycline to induce KSHV lytic replication $48 \mathrm{~h}$ prior to cell lysis. Data are presented as mean of technical duplicates. Error bars represent SD. Experiments were performed in biological duplicates, only one replicate shown.

present, albeit at different quantities, in both phases of the KSHV infectious cycle. Due to the repetitive nature of the TR (see Supplementary Table 5), qPCR was not suitable for measuring the specificity of R-loop precipitation in this region of the KSHV genome. However, traditional PCR clearly demonstrated the presence of TR sequences in samples immunoprecipitated with S9.6 antibody, while PCR amplification was not successful following precipitation with control Immunoglobulin G (IgG) (Fig. 4C). Moreover, treatment with RNase H resulted in the loss of S9.6 precipitation for TR sequences. Together, these results demonstrate that the RLFS prediction algorithm successfully identified bona fide RLFS in KSHV.

\section{Discussion}

Computational prediction is a useful tool to identify possible R-loop and RNA-DNA hybrid forming loci. QmRLFS-finder is powerful in identifying known R-loops in many cellular organisms which are universally coded by dsDNA genomes ${ }^{22,26}$. In this study, for the first time, we use QmRLFS-finder to predict R-loop loci in the genomes of remarkably versatile viral families. To perform R-loop mapping and comprehensive analysis, only complete viral genomes were explored. Initially, we considered excluding genomes of RNA viruses that do not require DNA in their life cycle. However, the need for information about the structure and stability of R-loops has recently arisen with the development of antisense oligonucleotides as novel antiviral and anticancer therapeutics ${ }^{39,40}$. Therefore, we included viral RNA genomes in our study because we believed that information about RNA-DNA hybrid loci could be useful for designing probes/oligos to bind or avoid R-loop forming regions. Thus, all types of viral genomes were utilized for R-loop prediction in this study.

Here, apart from computational prediction, we also experimentally demonstrated the presence of R-loops in KSHV genomes. Since the function of RLFS in viral genomes is essentially undefined, with only a single experimental evaluation, this resource highlights a number of molecular elements that could be readily targeted using $\mathrm{BAC}$ mutagenesis to define their functions in regulating gene expression, genome organization, and ultimately 
pathogenesis. For instance, since the EBV origin of replication consists of RLFS, we hypothesize that the RLFS in the KSHV TR are similarly involved in replication of the latent viral episome. Interestingly, ChIP-seq for LANA binding sites revealed overlap with several predicted RLFS in the host genome (examples in Supplementary Fig. 6), suggesting that R-loops may serve as docking sites for LANA. Moreover, given the difficulty of defining R-loop function in cellular biology, it is worth noting that the compact nature of herpesvirus genomes and ease of mutagenesis makes these viruses useful tools for further defining R-loop functions in various cellular biology. This finding also opens an opportunity to study viral-host interaction via R-loop structure. Future observations that explore R-loop occurring in vivo may provide information on regulation of viral phases. The predicted RLFS in other viral genomes provide potential links to further understanding the mechanisms that regulate their infectious cycles.

While a previous study focused on the relationship between R-loop and genome instability of host cell ${ }^{25}$, this study showcases widespread R-loop formation in multiple viral genomes, including KSHV. R-loop formation during KSHV infection influences the host transcription DNA damage response (DDR) and genome instability ${ }^{25}$. The KSHV ORF57 protein hijacks the host transcription and export complex (hTREX) protein, an RNA binding protein that prevents R-loop formation. This induces DNA damage in KSHV infected cells ${ }^{25}$. We hypothesize that hijacking hTREX protein from host cells creates an environment for increasing R-loop formation in the KSHV genome.

While RLFSs are a common sequence feature in all members in Herpesvirales, it is interesting that no RLFS was predicted in any members of Ligamenvirales. Ligamenvirales are classified into two families, Rudiviridae and Lipothrixviridae. Both families consist of virus with linear dsDNA genomes. These viruses infect archaea found in extreme environments ${ }^{41}$. Previous studies have showed that all members in Rudiviridae have a non-G-rich motif, AATTTAGGAATTTAGGAATTT, as a common sequence feature at the termini of their linear genomes. This motif can form DNA hairpin structures which contribute to DNA replication ${ }^{42}$, precluding the need for R-loops for initial strand separation and loading of core replication proteins as seen in $\mathrm{EBV}^{24}$. No hairpin structures are found the termini of Lipothrixviridae genomes, however, the study of this virus family is still incipient and somewhat limited.

Results generated from our analyses drove us to explore the RLFSs in herpesvirus genomes, however, there are several other virus families that contain high ratio of RLFS-positive genomes. For example, tymoviridae, which cause severe agricultural losses in many parts of the world. There are no studies indicating R-loop formation in these viruses. However, there is an increasing number of reports on R-loop formation in plants, the natural host of tymovirida $e^{43,44}$. RLFS data could be useful in guiding experimental efforts in global analysis of virus-host interactions in agriculturally important plants and their viral pathogens. This study presents a preliminary effort to explore the prevalence of R-loop in viral genomes. The generated RLFS prediction dataset can be used in a variety of dimensions, including in-depth investigation of the role of R-loops in host-pathogen interactions and manipulation of cellular processes by viruses.

Received: 18 November 2019; Accepted: 20 March 2020;

Published online: 14 April 2020

\section{References}

1. Nordheim, A. \& Rich, A. Negatively supercoiled simian virus 40 DNA contains Z-DNA segments within transcriptional enhancer sequences. Nature 303, 674-679 (1983).

2. McCarty, D. M., Young, S. M. Jr. \& Samulski, R. J. Integration of adeno-associated virus (AAV) and recombinant AAV vectors. Annu Rev Genet 38, 819-845, https://doi.org/10.1146/annurev.genet.37.110801.143717 (2004).

3. Schnepp, B. C., Clark, K. R., Klemanski, D. L., Pacak, C. A. \& Johnson, P. R. Genetic fate of recombinant adeno-associated virus vector genomes in muscle. J Virol 77, 3495-3504 (2003).

4. Metifiot, M., Amrane, S., Litvak, S. \& Andreola, M. L. G-quadruplexes in viruses: function and potential therapeutic applications. Nucleic Acids Res 42, 12352-12366, https://doi.org/10.1093/nar/gku999 (2014).

5. Dethoff, E. A. et al. Pervasive tertiary structure in the dengue virus RNA genome. Proc Natl Acad Sci USA, https://doi.org/10.1073/ pnas.1716689115 (2018).

6. Roy, D. \& Lieber, M. R. G clustering is important for the initiation of transcription-induced R-loops in vitro, whereas high G density without clustering is sufficient thereafter. Mol Cell Biol 29, 3124-3133, https://doi.org/10.1128/MCB.00139-09 (2009).

7. Roy, D., Yu, K. \& Lieber, M. R. Mechanism of R-loop formation at immunoglobulin class switch sequences. Mol Cell Biol 28, 50-60, https://doi.org/10.1128/MCB.01251-07 (2008)

8. Chedin, F. Nascent Connections: R-Loops and Chromatin Patterning. Trends Genet 32, 828-838, https://doi.org/10.1016/j. tig.2016.10.002 (2016).

9. Graf, M. et al. Telomere Length Determines TERRA and R-Loop Regulation through the Cell Cycle. Cell 170, 72-85 e14, https://doi. org/10.1016/j.cell.2017.06.006 (2017).

10. Kuznetsov, V. A., Bondarenko, V., Wongsurawat, T., Yenamandra, S. P. \& Jenjaroenpun, P. Toward predictive R-loop computational biology: genome-scale prediction of R-loops reveals their association with complex promoter structures, G-quadruplexes and transcriptionally active enhancers. Nucleic Acids Res, https://doi.org/10.1093/nar/gky690 (2018).

11. Wongsurawat, T., Jenjaroenpun, P., Kwoh, C. K. \& Kuznetsov, V. Quantitative model of R-loop forming structures reveals a novel level of RNA-DNA interactome complexity. Nucleic Acids Res 40, e16, https://doi.org/10.1093/nar/gkr1075 (2012).

12. Ginno, P. A., Lott, P. L., Christensen, H. C., Korf, I. \& Chedin, F. R-loop formation is a distinctive characteristic of unmethylated human CpG island promoters. Mol Cell 45, 814-825, https://doi.org/10.1016/j.molcel.2012.01.017 (2012).

13. Powell, W. T. et al. R-loop formation at Snord116 mediates topotecan inhibition of Ube3a-antisense and allele-specific chromatin decondensation. Proc Natl Acad Sci USA 110, 13938-13943, https://doi.org/10.1073/pnas.1305426110 (2013).

14. Yeo, A. J. et al. R-Loops in Proliferating Cells but Not in the Brain: Implications for AOA2 and Other Autosomal Recessive Ataxias. PLoS One 9, e90219, https://doi.org/10.1371/journal.pone.0090219 (2014).

15. Haeusler, A. R. et al. C9orf72 nucleotide repeat structures initiate molecular cascades of disease. Nature 507, 195-200, https://doi. org/10.1038/nature13124 (2014).

16. Kannan, A., Jiang, X., He, L., Ahmad, S. \& Gangwani, L. ZPR1 prevents R-loop accumulation, upregulates SMN2 expression and rescues spinal muscular atrophy. Brain 143, 69-93, https://doi.org/10.1093/brain/awz373 (2020). 
17. Kannan, A., Bhatia, K., Branzei, D. \& Gangwani, L. Combined deficiency of Senataxin and DNA-PKcs causes DNA damage accumulation and neurodegeneration in spinal muscular atrophy. Nucleic Acids Res 46, 8326-8346, https://doi.org/10.1093/nar/ gky641 (2018).

18. Perego, M. G. L., Taiana, M., Bresolin, N., Comi, G. P. \& Corti, S. R-Loops in Motor Neuron Diseases. Mol Neurobiol 56, 2579-2589, https://doi.org/10.1007/s12035-018-1246-y (2019).

19. Yang, Y. et al. Arginine methylation facilitates the recruitment of TOP3B to chromatin to prevent R loop accumulation. Mol Cell 53, 484-497, https://doi.org/10.1016/j.molcel.2014.01.011 (2014).

20. Garcia-Muse, T. \& Aguilera, A. R. Loops: From Physiological to Pathological Roles. Cell 179, 604-618, https://doi.org/10.1016/j. cell.2019.08.055 (2019).

21. Ginno, P. A., Lim, Y. W., Lott, P. L., Korf, I. \& Chedin, F. GC skew at the $5^{\prime}$ and $3^{\prime}$ ends of human genes links R-loop formation to epigenetic regulation and transcription termination. Genome Res 23, 1590-1600, https://doi.org/10.1101/gr.158436.113 (2013).

22. Jenjaroenpun, P., Wongsurawat, T., Sutheeworapong, S. \& Kuznetsov, V. A. R-loopDB: a database for R-loop forming sequences (RLFS) and R-loops. Nucleic Acids Res 45, D119-D127, https://doi.org/10.1093/nar/gkw1054 (2017).

23. Sanz, L. A. et al. Prevalent, Dynamic, and Conserved R-Loop Structures Associate with Specific Epigenomic Signatures in Mammals. Mol Cell 63, 167-178, https://doi.org/10.1016/j.molcel.2016.05.032 (2016).

24. Rennekamp, A. J. \& Lieberman, P. M. Initiation of Epstein-Barr virus lytic replication requires transcription and the formation of a stable RNA-DNA hybrid molecule at OriLyt. J Virol 85, 2837-2850, https://doi.org/10.1128/JVI.02175-10 (2011).

25. Jackson, B. R., Noerenberg, M. \& Whitehouse, A. A novel mechanism inducing genome instability in Kaposi's sarcoma-associated herpesvirus infected cells. PLoS Pathog 10, e1004098, https://doi.org/10.1371/journal.ppat.1004098 (2014).

26. Jenjaroenpun, P., Wongsurawat, T., Yenamandra, S. P. \& Kuznetsov, V. A. QmRLFS-finder: a model, web server and stand-alone tool for prediction and analysis of R-loop forming sequences. Nucleic Acids Res 43, 10081, https://doi.org/10.1093/nar/gkv974 (2015).

27. Zhang, Q., Jun, S. R., Leuze, M., Ussery, D. \& Nookaew, I. Viral Phylogenomics Using an Alignment-Free Method: A Three-Step Approach to Determine Optimal Length of k-mer. Sci Rep 7, 40712, https://doi.org/10.1038/srep40712 (2017).

28. Federhen, S. The NCBI Taxonomy database. Nucleic Acids Res 40, D136-143, https://doi.org/10.1093/nar/gkr1178 (2012).

29. Quinlan, A. R. BEDTools: The Swiss-Army Tool for Genome Feature Analysis. Curr Protoc Bioinformatics 47(11 12), 11-34, https:// doi.org/10.1002/0471250953.bi1112s47 (2014).

30. Brulois, K. F. et al. Construction and manipulation of a new Kaposi's sarcoma-associated herpesvirus bacterial artificial chromosome clone. J Virol 86, 9708-9720, https://doi.org/10.1128/JVI.01019-12 (2012).

31. Halasz, L. et al. RNA-DNA hybrid (R-loop) immunoprecipitation mapping: an analytical workflow to evaluate inherent biases. Genome Res 27, 1063-1073, https://doi.org/10.1101/gr.219394.116 (2017).

32. Ye, J. et al. Primer-BLAST: a tool to design target-specific primers for polymerase chain reaction. BMC Bioinformatics 13, 134, https://doi.org/10.1186/1471-2105-13-134 (2012).

33. Rigby, R. E. et al. RNA:DNA hybrids are a novel molecular pattern sensed by TLR9. EMBO J 33, 542-558, https://doi.org/10.1002/ embj.201386117 (2014)

34. Deng, Z., Wang, Z. \& Lieberman, P. M. Telomeres and viruses: common themes of genome maintenance. Front Oncol 2, 201, https:// doi.org/10.3389/fonc.2012.00201 (2012).

35. Yu, K., Chedin, F., Hsieh, C. L., Wilson, T. E. \& Lieber, M. R. R-loops at immunoglobulin class switch regions in the chromosomes of stimulated B cells. Nat Immunol 4, 442-451, https://doi.org/10.1038/ni919 (2003).

36. Kaufer, B. B., Jarosinski, K. W. \& Osterrieder, N. Herpesvirus telomeric repeats facilitate genomic integration into host telomeres and mobilization of viral DNA during reactivation. J Exp Med 208, 605-615, https://doi.org/10.1084/jem.20101402 (2011).

37. Arbuckle, J. H. \& Medveczky, P. G. The molecular biology of human herpesvirus-6 latency and telomere integration. Microbes Infect 13, 731-741, https://doi.org/10.1016/j.micinf.2011.03.006 (2011).

38. Toubiana, S. \& Selig, S. DNA:RNA hybrids at telomeres - when it is better to be out of the (R) loop. FEBS J 285, 2552-2566, https:// doi.org/10.1111/febs.14464 (2018)

39. Liang, X. H., Sun, H., Nichols, J. G. \& Crooke, S. T. RNase H1-Dependent Antisense Oligonucleotides Are Robustly Active in Directing RNA Cleavage in Both the Cytoplasm and the Nucleus. Mol Ther 25, 2075-2092, https://doi.org/10.1016/j. ymthe.2017.06.002 (2017)

40. Nakamori, M., Gourdon, G. \& Thornton, C. A. Stabilization of expanded (CTG)*(CAG) repeats by antisense oligonucleotides. Mol Ther 19, 2222-2227, https://doi.org/10.1038/mt.2011.191 (2011).

41. Prangishvili, D. \& Krupovic, M. A new proposed taxon for double-stranded DNA viruses, the order "Ligamenvirales". Arch Virol 157, 791-795, https://doi.org/10.1007/s00705-012-1229-7 (2012).

42. Vestergaard, G. et al. A novel rudivirus, ARV1, of the hyperthermophilic archaeal genus Acidianus. Virology 336, 83-92, https://doi. org/10.1016/j.virol.2005.02.025 (2005)

43. Xu, W. et al. The R-loop is a common chromatin feature of the Arabidopsis genome. Nat Plants, https://doi.org/10.1038/s41477-0170004-x (2017).

44. Sun, Q., Csorba, T., Skourti-Stathaki, K., Proudfoot, N. J. \& Dean, C. R-loop stabilization represses antisense transcription at the Arabidopsis FLC locus. Science 340, 619-621, https://doi.org/10.1126/science.1234848 (2013).

\section{Acknowledgements}

We thank Dr. David W Ussery for his comments and suggestions, Mr. Preecha Patumcharoenpol for sharing viral genome data and Dr. Srijon Kaushik Banerjee for lab technical assistance. This work was funded in part by the National Institute of General Medical Sciences of the National Institutes of Health award number P20GM125503 and the National Cancer Institute of the National Institutes of Health award number R01-CA167065.

\section{Author contributions}

I.N. and T.W. designed and conceived the project. I.N., J.C.F. supervised the study. A.G. and S.O. performed experiments. A.G., T.W. and J.C.F. interpreted experimental results. P.J. and T.W. performed computational analysis. I.N. and P.J. performed data visualization. T.W. and I.N. structured the manuscript. T.W. drafted the manuscript. All authors participated in interpreting the results and writing the manuscript, and read and approved the final version of this manuscript.

\section{Competing interests}

The authors declare no competing interests.

\section{Additional information}

Supplementary information is available for this paper at https://doi.org/10.1038/s41598-020-63101-9.

Correspondence and requests for materials should be addressed to T.W. or I.N. 
Reprints and permissions information is available at www.nature.com/reprints.

Publisher's note Springer Nature remains neutral with regard to jurisdictional claims in published maps and institutional affiliations.

(c) (i) Open Access This article is licensed under a Creative Commons Attribution 4.0 International License, which permits use, sharing, adaptation, distribution and reproduction in any medium or format, as long as you give appropriate credit to the original author(s) and the source, provide a link to the Creative Commons license, and indicate if changes were made. The images or other third party material in this article are included in the article's Creative Commons license, unless indicated otherwise in a credit line to the material. If material is not included in the article's Creative Commons license and your intended use is not permitted by statutory regulation or exceeds the permitted use, you will need to obtain permission directly from the copyright holder. To view a copy of this license, visit http://creativecommons.org/licenses/by/4.0/.

(C) The Author(s) 2020 